\title{
EVALUATION OF PATIENTS' AWARENESS REGARDING GLUCOCORTICOIDS SIDE EFFECTS AND AVAILABLE PREVENTION STRATEGIES
}

\author{
Cristina Plaisanu ${ }^{1}$, Vasilia Cristina Iorgoveanu ${ }^{2}$, Ruxandra Ionescu ${ }^{1,2}$ \\ ${ }^{I}$ Carol Davila University of Medicine and Pharmacy, Bucharest \\ ${ }^{2}$ Department of Internal Medicine and Rheumatology, Sf. Maria Clinical Hospital, Bucharest
}

\begin{abstract}
Glucocorticoids are drugs used in various medical areas, but well known for the myriad of side effects which are still a concern for the medical world. Latest recommendations on this matter aim at improving their management and implementing adequate prevention strategies. This aspect would change patient's perspective on this treatment, reducing the fear and uncertainty associated with glucocorticoids.

Objective. Our study focuses on analysing the level of information that patients hold about glucocorticoids side effects and if the available prevention strategies are known and applied in clinical practice.

Material and methods. Study included 85 patients with rheumatic pathology and glucocorticoid therapy for at least 3 months. Study design was prospective observational and consecutive hospitalized patients were recruited, receiving and compiling a specific questionnaire.

Results. 85 patients ( $84.7 \%$ women, mean age $54.42 \pm 16.54$ years) mostly under corticotherapy due to rheumatoid arthritis (44.7\%) and systemic lupus erythematosus $(23.5 \%)$ were recruited. Patients with higher education had the tendency to discontinue treatment $(r=0.289, p=0.007)$. Moreover, reading prospectus has a great influence on treatment compliance (treatment risks significantly correlated with disease duration and glucocorticoid therapy duration ( $r=0.232 ; p<0.032, r=0.252 ; p<0.024$ respectively). Awareness about the risk of osteoporosis correlates with reading the prospectus $(r=0.396 ; p<0.0001)$, glucocorticoid treatment duration $(r=0.209 ; p<0.032)$ and the use of vitamin D supplements ( $r=0,315$; $p<0.003)$. Regarding glucocorticoid administration timetable, we identified two groups - early morning and late evening (44.7\% vs. 55.3\%), showing a non-uniform treatment schedule. Conclusions. Our study shows an inadequate level of information regarding patient's awareness on glucocorticoid treatment. Although excellent prevention strategies have been elaborated, there is a need of optimizing their accessibility to our patients.
\end{abstract}

Keywords: glucocorticoids, side effects, prevention strategies

\section{INTRODUCTION}

The use of glucocorticoids (GC) represents a vital element for rheumatic pathology management. Various side effects generate a lower patient compliance, increased morbidity and enormous costs for health services. Glucocorticoids can solve critical medical situations by the use of high doses on short duration but are more frequently used as low/medium doses and long-term therapy (1). Side effects such as iatrogenic Cushing syndrome, electrolyte disturbances, osteoporosis and vertebral fractures, glucose intolerance, cataracts, glaucoma, myopathy, psychiatric and digestive disorders seem to be proportional to treatment dosage and duration (2). Recent data sug- gest that also low doses $<7.5 \mathrm{mg}$ prednisone equivalent/day can generate important side effects and are not safer than other treatment regimens $(3,4)$.

In 2013 EULAR task force on glucocorticoids discussed and created a series of recommendations on patients and doctors' education, aiming to improve knowledge by implementing preventive measures, optimizing treatment regimens and adequately monitoring side effects (5). All recommendations were based on complex analysis of existing studies and on evidence derived from clinical practice but also taking into account patients opinion and preferences. This shows, once again, the need for patient involvement in treatment optimization and that, in order to diminish negative treatment implications, 
we have to create a doctor-patient team focused on an adequately informed patient.

Our study identified whether patients were explained and understood therapy risks and purpose, if they abruptly discontinued treatment on own initiative and if they know and have experienced unwanted effects. We also evaluated the accessibility of latest prevention strategies such as avoiding smoking and alcohol, performing weight bearing exercise, annual eye examination and osteodensitometry, etc. Study goal was not only to analyse a situation but also to set a warning signal regarding the fact that, although good theoretical strategies exist, the key of their proper real-life implementation is a correctly informed and actively involved patient.

Therefore, our study had the purpose to accomplish the following main objectives:

- Identification of glucocorticoid treatment indication, administration hours, rheumatic pathology duration and glucocorticoid treatment length;

- Analysing patient's education regarding glucocorticoids and doctor-patient communication treatment aim and potential risks have been understood by the patients and explained by their physicians;

- Analysing patient's awareness regarding life style modification in order to diminish potential side effects - diet improvement, alcohol and smoking avoidance, vitamin D and calcium supplementation, regular exercise in order to prevent obesity, reduce cardiovascular risk, prevent muscular side effects and osteoporosis;

- Identification of appropriate preventive/therapeutic interventions implemented by doctors for a good clinical practice regarding the risk of glucocorticoid induced osteoporosis, ocular complications, and hypothalamic-pituitary-adrenal axis suppression;

- Assessment of patient's information modalities, treatment prospectus understanding and reading.

\section{MATERIALS AND METHODS}

The study was conducted in the Internal Medicine and Rheumatology Department of Sfanta Maria Clinical Hospital, from January to December 2014. Study design was prospective observational and consecutive hospitalized patients were recruited, over 18 years of age with indication of long-term oral glucocorticoid treatment ( $>3$ months). Most frequently rheumatoid arthritis, systemic lupus erythematosus, polymyalgia and other vasculitides, poly/ dermatomyositis, systemic sclerosis, mixed connec- tive tissue disease were included in the pathology. Patients signed an informed consent explaining study purpose and patient's involvement details. Study respected patient's data confidentiality and had the agreement of local Ethics Committee. Study involved the development of a special questionnaire who was self-completed by the patient and included demography and educational data, medical data, assessment of knowledge level, assessment of prevention strategies implementation and knowledge. The questionnaire used patient accessible terminology and all patients were able to communicate with the investigator if necessary. The questionnaire included 13 items regarding treatment awareness, main aspects are listed below:

- Awareness on glucocorticoids treatment risks/ purpose;

- Awareness on glucocorticoid osteoporosis risk;

- Awareness on the risk on muscular side effects;

- Awareness on the need for weight bearing activities;

- Awareness on the risk of adrenal axis suppression;

- Awareness on the need to avoid smoking and alcohol consumption;

- Awareness on the risk of ophthalmic complications;

- Awareness on the risk of psychiatric effects;

- Awareness on the risk of weight gaining;

- Reading glucocorticoid treatment leaflet.

We also analysed implementation of available side effects prevention strategies such as DXA testing, ophthalmic complications screening, vitamin D supplementation, and bisphosphonate treatment. Obtained data were statistical evaluated using IBM SPSS Statistics program version 20.0 for Windows. Before data processing, we analysed them for normal Gaussian distribution in order to report them as means with standard deviation or median. Differences between types of data were assessed using non - parametric binomial test and statistical significance was determined by Spearman correlation coefficients using two-sided tests, considered significant at $\mathrm{p} \leq 0.05$.

\section{RESULTS}

We evaluated and validated 85 questionnaires during the study protocol. Study included 72/85 $(84.7 \%)$ women, mean age $54.42 \pm 16.54$ years, of 
which 60/85 (70.6\%) had secondary education whilst only $25 / 85$ patients (29.4) had university education. Regarding rheumatic illnesses encountered in the study group, there were $38 / 85$ (44.7\%) patients with rheumatoid arthritis, 20/85 (23.5\%) systemic lupus erythematosus, and the remaining 27/85 (31.8\%) had other rheumatic conditions as will be detailed in tables below (Table 1 and Table 2).

TABLE 1. Study group characteristics $(N=85)$

\begin{tabular}{|l|c|c|}
\hline \multicolumn{1}{|c|}{ Characteristic } & Number & Percentage $\%$ \\
\hline Men & 13 & 15.3 \\
\hline Women & 72 & 84.7 \\
\hline Age (years) - mean \pm SD & $54.42 \pm 16.54$ & \\
\hline Age groups & & \\
- 18-29 years & 8 & 9.4 \\
- 30-49 years & 22 & 25.9 \\
- 50-69 years & 41 & 48.2 \\
- 70-87 years & 14 & 16.5 \\
\hline Education & & \\
- High school/gymnasium & 60 & 70.6 \\
- University education & 25 & 29.4 \\
\hline
\end{tabular}

Median disease duration amongst patients was 72 months $95 \%$ CI $(80.9$ - 123.7) and median glucocorticoid therapy duration was 42 months $95 \%$ CI (54.1 - 86.5). Regarding glucocorticoid administration timetable, we noticed two types of preferences amongst patients: $38 / 85$ patients $(44.7 \%)$ are taking the drug between 1:00-12:00 AM and the remaining 47/85 (55.3\%) administer the cortisone in the afternoon from 13:00-24:00 PM. Two peaks were observed in terms of administration: the hours of 8:00 to 9:00 AM for $13 / 85(15.3 \%)$ and the hours $10: 00$ to 11:00 PM for $16 / 85(18.8 \%)$, with the remaining hour's frequencies below 2-5\% for each other hour.

TABLE 2. Detailed medical data of study group $(N=85)$

\begin{tabular}{|l|c|c|}
\hline \multicolumn{1}{|c|}{ Characteristic } & Number & Percentage \% \\
\hline Diagnostic & & \\
- Rheumatoid arthritis & 38 & 44.7 \\
- Systemic lupus erythematosus & 20 & 23.5 \\
- Vasculitides & 6 & 7.0 \\
- Poli/dermatomyositis & 5 & 5.8 \\
- Systemic sclerosis & 5 & 5.8 \\
- Others & 11 & 13.2 \\
\hline Median disease duration (months) & $72(3-408)$ & \\
\hline Median glucocorticoid therapy & $42(3-360)$ & \\
duration (months) & & \\
\hline Administration hours intervals & & \\
- 1:00-12:00 AM & 38 & 44.7 \\
- 13:00-24:00 PM & 47 & 55.3 \\
\hline
\end{tabular}

Our evaluation showed that $21 / 85$ (24.7\%) patients have changed on own initiative their daily treatment doses and 33/85 (38.8\%) patients have abruptly discontinued glucocorticoid therapy without a doctor's indication. There was a statistically significant correlation between dose modification/ discontinuation and patients with university education $(\mathrm{r}=0.289 ; \mathrm{p}<0.007, \mathrm{r}=0.280 ; \mathrm{p}<=0.009$ respectively). We did not obtain significant correlations between changing glucocorticoid doses/ interrupting treatment and certain age groups, sex, diagnoses, hours of administration. However, reading treatment prospectus correlated significantly with therapy discontinuation without the doctor's agreement $(r=0.267 ; p<0.014)$ and therapy duration significantly correlated with the tendency to stop treatment $(\mathrm{r}=0.239 ; \mathrm{p}=0.035)$.

Regarding awareness on treatment goals and risks, we observed that $41 / 85$ (48.2\%) patients consider they have been informed on treatment purpose and only $28 / 85(32.9 \%)$ felt that the associated risks were explained. Reading treatment prospectus significantly correlated with awareness on treatment risks $(\mathrm{r}=0.357$; $\mathrm{p}<0.001)$ and information on treatment risks significantly correlated with disease duration and glucocorticoid therapy duration $(\mathrm{r}=0.232 ; \mathrm{p}<0.032, \mathrm{r}=0.252 ; \mathrm{p}$ $<0.024$ respectively). A highly significant statistical correlation was observed between awareness on treatment goals/risks and awareness on the need to not abruptly discontinue treatment $(\mathrm{r}=0.507 ; \mathrm{p}<0.0001)$. In Table 3, presented below, we summarize the evaluation of the level of information in the study group.

TABLE 3. Evaluation of patient information on glucocorticoid therapy $(N=85)$

\begin{tabular}{|l|c|c|}
\hline \multicolumn{1}{|c|}{ Characteristic } & Number & Percentage \% \\
\hline Awareness on treatment purpose & 41 & 48.2 \\
\hline Awareness on treatment risks & 28 & 32.9 \\
\hline $\begin{array}{l}\text { Awareness on glucocorticoid induced } \\
\text { osteoporosis }\end{array}$ & 51 & 60 \\
\hline Awareness on muscular side effects & 39 & 45.9 \\
\hline $\begin{array}{l}\text { Awareness on the need for weight } \\
\text { bearing activities }\end{array}$ & 20 & 23.5 \\
\hline $\begin{array}{l}\text { Awareness on not to abruptly } \\
\text { discontinue treatment }\end{array}$ & 53 & 62.4 \\
\hline $\begin{array}{l}\text { Awareness on the risk of adrenal axis } \\
\text { suppression }\end{array}$ & 27 & 31.8 \\
\hline $\begin{array}{l}\text { Awareness on the need to avoid } \\
\text { smoking }\end{array}$ & 31 & 36.5 \\
\hline $\begin{array}{l}\text { Awareness on the need to avoid } \\
\text { alcohol }\end{array}$ & 62 & 72.9 \\
\hline $\begin{array}{l}\text { Awareness on the risk of ophthalmic } \\
\text { complications }\end{array}$ & 43 & 50.6 \\
\hline $\begin{array}{l}\text { Awareness on the risk of psychiatric } \\
\text { effects }\end{array}$ & 34 & 40 \\
\hline $\begin{array}{l}\text { Awareness on the risk of weight } \\
\text { gaining }\end{array}$ & 23 & 27.1 \\
\hline $\begin{array}{l}\text { Reading glucocorticoid treatment } \\
\text { leaflet }\end{array}$ & 59 & 69.4 \\
\hline
\end{tabular}


Awareness about the risk of osteoporosis did not correlate with age, sex, diagnosis, level of education or DXA testing, but there was a significant correlation with reading the prospectus $(\mathrm{r}=0.396 ; \mathrm{p}<0.0001)$, glucocorticoid treatment duration $(\mathrm{r}=0.209 ; \mathrm{p}<0.032)$ and the use of vitamin D supplements $(r=0,315$; $\mathrm{p}<0.003)$.

TABLE 4. Evaluation of glucocorticoid side effects prevention strategies implementation $(N=85)$

\begin{tabular}{|l|c|c|}
\hline \multicolumn{1}{|c|}{ Characteristic } & Number & Percentage \% \\
\hline Osteodensitometry testing & 40 & 47.1 \\
\hline $\begin{array}{l}\text { Cardiovascular risk reduction through } \\
\text { smoking avoidance }\end{array}$ & 31 & 36.5 \\
\hline $\begin{array}{l}\text { Cardiovascular risk reduction through } \\
\text { alcohol avoidance }\end{array}$ & 62 & 72.9 \\
\hline $\begin{array}{l}\text { Myopathy/osteoporosis/obesity } \\
\text { prevention through weight-bearing } \\
\text { exercise, muscle training }\end{array}$ & 20 & 23.5 \\
\hline Ophthalmologic evaluation & 67 & 78.8 \\
\hline Vitamin D supplementation & 70 & 82.4 \\
\hline Calcium supplementation & 15 & 17.6 \\
\hline Bisphosphonates treatment & 22 & 25.9 \\
\hline $\begin{array}{l}\text { Hypothalamic-pituitary-adrenal axis } \\
\text { suppression prevention through correct } \\
\text { administration/dose tapering }\end{array}$ & 52 & 61.2 \\
\hline
\end{tabular}

Osteodensitometry testing significantly correlated with age, disease duration and duration of glucocorticoid therapy $(p=0.0001)$ but did not significantly correlate with education level, reading treatment prospectus or the rheumatic disease. Regarding osteoporosis screening through DXA testing, 20/38 (52.6\%) patients with rheumatoid arthritis were performed a DXA testing, 4/20 (20\%) of patients with systemic lupus erythematosus and 16/27 (59.2\%) patients with other diseases. Ophthalmologic exam was performed on $28 / 38$ (73.6\%) rheumatoid arthritis patients, $17 / 20(85 \%)$ systemic lupus erythematosus and 22/27 (81.4\%) other diseases patients.

\section{DISCUSSION}

The main objective of this study was to assess the level of awareness on glucocorticoid side effects amongst rheumatic patients. Our intention was to analyse if such a highly used treatment is also properly perceived through a good doctor-patient communication and if prevention strategies are optimally implemented in our medical practice. It should be noted that, although a significant percentage of patients reported having read treatment prospectus (69.4\%), this didn't generate an adequate level of awareness on glucocorticoids. Medical language and the amount of information could interfere with patient's ability to understand his treatment and also to apply side effects prevention strategies.

On the contrary, reading treatment prospectus has generated a tendency to stop the treatment without the doctor's advice, this trend being more important among patients with higher education. University education patients are more likely to seek various information sources, more or less agreed by the medical world. This aspect may cause interference with treatment dose/duration and decreased compliance to recommendations. In this regard, physicians should pay more time and attention at explaining treatment risks and purpose, especially to higher education patients, and, as much as possible, providing them reliable additional information sources.

Our study revealed that there is an inconsistency regarding glucocorticoids administration hours, significant percentages of patients have preference for 8-9:00 AM (15.3\%) or 22-23:00 PM (18.8\%), respectively. In order to minimize the risk of adrenal insufficiency, glucocorticoids should be administered early in the morning. On the other hand, to have an ideal rheumatic effect, particularly joint morning stiffness, a late evening administration is recommended in our practice. We can say that there is an obvious need to establish clear administration hours, taking into account that administration schedules can definitely affect side effects profile and also patient's compliance. Currently glucocorticoid chronobiology represents a novel research subject with promising clinical implications (6).

A relatively small percentage of patients considered themselves properly informed about treatment risks and objectives $-48.2 \%$ and $32.9 \%$ respectively. Obviously, elderly patients with longer disease duration were better informed on this subject. Many patients change or discontinue treatment without physician's advice, this showing the need to improve patient-doctor communication. This deficiency could be improved by modern medical solutions such as telemedicine, mostly unavailable at the moment in our practice.

Managing glucocorticoids side effects, especially osteoporosis and osteoporotic fractures, involves high financial costs, as various studies reveal (7-9) and various prevention strategies have been promoted (10-12). Our study shows that approximately $47 \%$ of patients have been performed a bone densitometry, this reflecting an improper screening of this complication in clinical practice. Patients were in- 
formed about the risk of glucocorticoid induced osteoporosis $(60 \%)$, although life style modification and sedentary attitude is still a problem for our patients. Latest EULAR and ACR recommendations $(5,13,14)$ are not properly known and applied, more efforts are needed in order to implement this prevention strategies starting from patients, patients' treatment teams, relatives and family physicians. Cardiovascular risk could be diminished through simple life style changes such as alcohol/smoking cessation and daily exercise in order to prevent obesity.

Regarding the abrupt cessation of treatment, we found that $62 \%$ of patients knew they should not abruptly stop glucocorticoid treatment, although the risk of adrenal suppression was known by only $32 \%$ of patients. This aspect is considered extremely important since it can cause serious complications, difficult to redress $(15,16)$. This highlights the need for additional explanations in order to increase compliance. Patients were explained and understood the risk of ophthalmic complications (50\%) and we noticed a good ocular screening in our department. We also noticed that majority of patients $(82 \%)$ had received vitamin D supplements, although calcium supplementation is diminished. Regarding glucocorticoid induced myopathy, evidence shows that training muscles for strength and performing weightbearing exercises on a daily basis (5) represents an essential and simple manner of preventing a redoubtable complications. Regrettably, sedentary attitude has an increased frequency amongst all age groups, although represents the most correctable aspect of all.

Although our study is limited due to sample size especially, to our knowledge, it is the first survey of these objectives. Our study results can have important educational implications regarding glucocorticoids side effects management.

\section{CONCLUSIONS}

The study shows insufficient awareness of glucocorticoid side effects and prevention strategies amongst patients. Although efforts have been made in order to promote prevention methods, there is an inappropriate knowledge and accessibility on this matter. This could be prevented by improving patient-doctor communication taking into account that optimizing communication would increase patient compliance and awareness on a highly used therapy. Patients can be compliant if properly informed and, if further steps are taken for their education, significant glucocorticoid risks would be prevented by simple methods related mainly to lifestyle modification.

We also consider more studies are needed in order to clarify the safest hour of administration for glucocorticoids, because timing certainly has a significant impact on treatment's risk profile. Because in real practice actual time spent with the patient is limited, treatment awareness could be improved with the help of reliable informative materials and a patient accessible language. Monitoring glucocorticoids side effects could benefit in the future from the latest technology of telemedicine, currently successfully applied to various other treatment strategies (17-19).

\section{Acknowledgement}

This work received financial support through the project entitled "CERO - Career profile: Romanian Researcher", grant number POSDRU/159/1.5/S/ 135760, cofinanced by the European Social Fund for Sectoral Operational Programme Human Resources Development 2007-2013.

5. Duru N., Van der Goes M.C., Jacobs J.W.G., Andrews T., Boers M. - EULAR evidence-based and consensus-based recommendations on the management of medium to high-dose glucocorticoid therapy in rheumatic diseases, Annals of the Rheumatic Diseases 2013, ard.bmj.com. Ann Rheum Dis 2013, doi:10.1136/annrheumdis-2013-203249

6. Buttgereit F., Da Silva J.A.P., Boers M. et al. - Standardised nomenclature for glucocorticoid dosages and glucocorticoid treatment regimens: current questions and tentative answers in rheumatology, Extended report. Ann Rheum Dis 2002; 61:718-722 doi:10.1136/ard.61.8.71 
7. Manson S.C., Brown R.E., Cerulli A., Vidaurre C.F. - The cumulative burden of oral corticosteroid side effects and the economic implications of steroid use, Respiratory Medicine, Volume 103, Issue 7, July 2009, Pages 975-994, doi:10.1016/j. rmed.2009.01.003

8. Veenstra D.L., Best J.H., Hornberger J., Sullivan S.D., Hricik D.E. - Incidence and long-term cost of steroid-related side effects after renal transplantation, Am J Kidney Dis. 1999 May; 33(5):829-39

9. Pisu M., James N., Sampsel S., Saag K.G. - The cost of glucocorticoid-associated adverse events in rheumatoid arthritis, Rheumatology, June 2005, 44(6):781-788.doi:10.1093/ rheumatology/keh594

10. Sadat-Ali M., Alelq A.H., Alshafei B.A., Al-Turki H.A., Abujubara M.A. - Osteoporosis prophylaxis in patients receiving chronic glucocorticoid therapy. Ann Saudi Med. 2009 MayJun; 29(3):215-8. PubMed PMID:19448373; PubMed Central PMCID:PMC2813647

11. Ledwich L.J., Clarke K. - Screening and treatment of glucocorticoid-induced osteoporosis in rheumatoid arthritis patients in an urban multispecialty practice. J Clin Rheumatol. 2009 Mar; 15(2):61-4. doi: 10.1097/RHU.0b013e31819b65bd. PubMed PMID: 19265346

12. Massafra U., Migliaccio S., Bancheri C., Chiacchiararelli F., Fantini F., Leoni F., Martin L.S., Migliore A., Muccifora B., Napolitano C., Pastore R., Ragno A., Ronzoni S., Rotondi M., Tibaldi M., Villa P., Vinicola V., D‘Erasmo E., Falaschi P., Minisola G. - Approach in glucocorticoid-induced osteoporosis prevention: results from the Italian multicenter observational EGEO study. J Endocrinol Invest. 2013 Feb; 36(2):92-6. doi:10.3275/8288. Epub 2012 Mar 6. PubMed PMID: 22398397
13. Moghadam-Kia S., Werth V.P. - Prevention and treatment of systemic glucocorticoid side effects. Int J Dermatol. 2010 Mar; 49(3):239-248. doi: 10.1111/j.1365-4632.2009.04322.x

14. Grossman J.M. et al. - American College of Rheumatology 2010 Recommendations for the Prevention and Treatment of Glucocorticoid-Induced Osteoporosis. Arthritis Care \& Research November 2010; 62(11):1515-1526. DOI 10.1002/acr.20295, 2010; American College of Rheumatology

15. Dinsen S., Baslund B., Klose M., Rasmussen A.K., Friis-Hansen L., Hilsted L., Feldt-Rasmussen U. - Why glucocorticoid withdrawal may sometimes be as dangerous as the treatment itself. Eur J Intern Med. 2013 Dec; 24(8):714-20 doi:10.1016/j.ejim.2013.05.014. Epub 2013 Jun 25. Review. Erratum in: Eur J Intern Med. 2014 Oct; 25(8):781-3. PubMed PMID: 23806261

16. Huber B.M., Bolt I.B., Sauvain M.J., Flück C.E. - Adrenal insufficiency after glucocorticoid withdrawal in children with rheumatic diseases. Acta Paediatr. 2010 Dec; 99(12):1889-93. doi: 10.1111/j.1651 2227.2010.01936.x. PubMed PMID:20649769

17. Testa S., Paoletti O., Zimmermann A., Bassi L., Zambelli S., Cancellieri E. - The Role of Anticoagulation Clinics in the Era of New Oral Anticoagulants. Thrombosis, vol. 2012, Article ID 835356, 6 pages, 2012. doi:10.1155/2012/835356

18. Jakobsen A.S., Laursen L.C., Østergaard B., Rydahl-Hansen S., Phanareth K.V. - Hospital-admitted COPD patients treated at home using telemedicine technology in The Virtual Hospital Trial: methods of a randomized effectiveness trial. Trials. 2013; 14:280. doi:10.1186/1745-6215-14-280

19. Bernocchi P., Scalvini S., Bertacchini F., Rivadossi F., Muiesan M.L. - Home based telemedicine intervention for patients with uncontrolled hypertension: - a real life - non-randomized study. BMC Med Inform Decis Mak. 2014; 14:52 